\title{
Developmental Expression of Monocarboxylate Transporter 1 and 4 in Rat Liver
}

\author{
Michael $\mathrm{Ng}^{1}$, Justin Louie ${ }^{1,2}$, Jieyun $\mathrm{Cao}^{1}$, and Melanie A. Felmlee ${ }^{1}$ \\ ${ }^{1}$ Department of Pharmaceutics and Medicinal Chemistry, Thomas J Long School of Pharmacy and Health Sciences, \\ University of the Pacific, Stockton, CA, USA; ${ }^{2}$ Dignity Health, Mercy General Hospital, Sacramento, CA, USA
}

Received, May 29, 2019; Revised, July 19, 2019; Accepted, July 20, 2019; Published, July 23, 2019.

\begin{abstract}
Purpose: Monocarboxylate transporters (MCT) are proton-coupled integral membrane proteins that control the influx and efflux of endogenous monocarboxylates such as lactate, acetate and pyruvate. They also transport and mediate the clearance of drugs such as valproate and gamma-hydroxybutyrate. CD147 functions as ancillary protein that chaperones MCT1 and MCT4 to the cell membrane. There is limited data on the maturation of MCT and CD147 expression in tissues related to drug distribution and clearance. The objective of the present study was to quantify hepatic MCT1, MCT4, and CD147 mRNA, whole cell and membrane protein expression from birth to sexual maturity. Methods: Liver tissues were collected from male and female Sprague Dawley rats at postnatal days (PND) $1,3,5,7,10,14,18,21,28,35$, and $42(\mathrm{n}=3-5)$. Hepatic mRNA, total and membrane protein expression of MCT1, MCT4, and CD147 was evaluated via qPCR and western blot. Results: MCT1 mRNA and protein demonstrated nonlinear maturation patterns. MCT1 and CD147 membrane protein exhibited low expression at birth, with expression increasing three-fold by PND14, followed by a decline in expression at sexual maturity. MCT4 mRNA had highest expression at PND 1, with decreasing expression towards sexual maturity. In contrast, MCT4 membrane protein exhibited minimal expression from birth through weaning before a 10-fold surge at PND35, whereupon there was a sharp decline in expression at PND42. There was a significant positive correlation between MCT1 and CD147 whole cell and membrane expression, while MCT4 membrane expression demonstrated a weak negative correlation with CD147. Conclusion: Our study elucidates the transcriptional and translational maturation patterns of MCT1, MCT4 and CD147 expression, with isoformdependent differences in the liver. Changes in transporter expression during development may greatly influence drug distribution and clearance in pediatric populations.
\end{abstract}

\section{INTRODUCTION}

Maturation of drug elimination pathways contributes to the wide range of inter-individual variability in drug disposition observed in pediatric populations. Clinical pharmacokinetic studies have identified post-natal age as a significant factor influencing clearance, with hepatic and renal elimination representing the primary mechanisms of drug clearance $(1,2)$. Developmental change in hepatic drug clearance are governed by maturation of metabolism and drug transport pathways. While maturation of drug metabolizing enzymes (3), and major hepatic drug transporters (4) have been extensively studied in multiple species, there is limited data evaluating the maturation of monocarboxylate transporters in the liver.

Monocarboxylate transporters are involved in the active transport of essential nutrients and pharmaceutical agents, including lactate, pyruvate, butyrate and ketone bodies, nicotinate, valproic acid, nateglinide, salicylate, atorvastatin, and $\gamma$ hydroxybutyrate (GHB) (5-7). There are two monocarboxylate transporter families, SLC16A and SLC5A; however, only proton-dependent monocarboxylate transporters (MCTs) encoded by SLC16A are expressed in the liver. There are 14 members in the MCT family with MCT1 through 4 (SLC16A1, SLC16A7, SLC16A8 and SLC16A3) involved in proton-coupled transport with overlapping substrate specificity $(5,8)$. MCT1 and 4 , the primary MCTs expressed in the liver, are sorted and inserted into the basolateral plasma membrane of hepatocytes through their association with the ancillary protein CD147 (9).

Corresponding Author: Dr. Melanie A. Felmlee, Department of Pharmaceutics and Medicinal Chemistry, Thomas J. Long School of Pharmacy and Health Sciences, University of the Pacific, Stockton, CA 95211, USA; Email: mfelmlee@pacific.edu 
MCTs function as bidirectional transporters with directional flux of MCT substrates governed by the $\mathrm{pH}$ gradient (10), with rate of transport also dependent on MCT and CD147 expression and colocalization (11). Changes in the regulation or trafficking of MCTs leading to differences in membrane expression can alter the hepatic transport of their substrates, leading to alterations in intracellular drug concentrations. Differences in hepatic transport may impact overall drug exposure, which can lead to toxic or sub-therapeutic drug levels during development (2). It is vital to understand the maturation of these transporters to optimize therapeutic regimens for their substrates.

There is limited information in the literature on the maturation of monocarboxylate transporters in tissues related to drug disposition. MCT1 has transient embryonic expression in the dorsal and lateral anlage of rat cerebral cortex (12), whereas in the gluteus medius muscle in horse, MCT1 increases and MCT4 is stably expressed with age (13), suggesting MCTs are regulated in a tissue-specific manner. MCT expression differs based on species, with minimal expression of MCT1 in hamster liver, whereas MCT1 is the predominant isoform expressed in mouse, rat, and human livers (12). Proteomic studies of human hepatic MCT1 show nonlinear, age-dependent levels of expression (13). These studies indicate that MCT maturation should be evaluated in individual tissues to identify tissuespecific maturation patterns of each isoform and the potential impact on drug pharmacokinetics. The objective of the present study is to quantify agedependent hepatic mRNA, total and membrane protein expression of MCTs and their ancillary protein CD147 in male and female rats, to evaluate their developmental regulation with respect to transcription, translation and membrane trafficking.

\section{METHODS}

Animals and Tissue Collection: All animal experiments were approved by the Institutional Animal Care and Use Committees of University at Buffalo (SUNY) and University of the Pacific. Male and female Sprague-Dawley rats ( $>21$ days of age), pregnant females (E14 on arrival) and lactating females with litters (post-natal day seven on arrival) were obtained from Envigo (USA). For pups less than 21 days of age, one male and one female pup was removed from each litter at individual post-natal time points. Pups were weaned at 21 days of age. Rats were group housed (except pregnant females) and kept under controlled lighting (12h light/dark cycle) and temperature $\left(20 \pm 2^{\circ} \mathrm{C}\right)$ conditions with food and water provided ad libitum. Liver samples were collected ( $\mathrm{N}=3$ - 5 per gender per age group) from post-natal day (PND) 1 through 42 following exsanguination under isoflurane anesthesia, and snap frozen in liquid nitrogen with storage at $-80^{\circ} \mathrm{C}$. Body and liver weights are detailed in Figure 1.

Sex Determination: Sex was assigned for pups less than PND21 by ano-genital distance at the time of tissue collection and was confirmed by PCR. Liver (25 mg) was utilized to isolate genomic DNA using a PureLink Genomic DNA Mini Kit (Invitrogen) following the manufacturer's instructions. DNA concentration and purity was evaluated using a Nanodrop (Thermo Scientific). The presence of SRY, a Y specific sequence, and $\beta$-actin (PCR control) were evaluated by end-point PCR following a previously published method with minor modifications (14). Briefly, $2 \mu \mathrm{l}$ of genomic DNA was combined with AmpliTaq Gold (ThermoFisher) and corresponding buffer, $0.2 \mathrm{mM}$ dNTPs, $0.2 \mu \mathrm{M}$ forward and reverse primers (Table 1) with a final reaction volume of $25 \mu \mathrm{l}$. PCR reactions were run separately for each gene. Samples were run through a thermal cycle using a T100 Thermal Cycler (BioRad) as follows: 1 minute at $95^{\circ} \mathrm{C}, 1$ minute at $52^{\circ} \mathrm{C}$, and 1 minute at $72^{\circ} \mathrm{C}$ with the cycle repeated 35 times. PCR products were separated on a $1.4 \%$ agarose gel containing SYBR Safe (Invitrogen) in $1 \mathrm{X}$ TBE buffer, run at $100 \mathrm{~V}$ for 45 minutes and visualized using ChemiDoc Touch imaging system (BioRad).

qPCR: Liver $(10 \mathrm{mg})$ was homogenized and RNA was isolated using a PureLink RNA Mini kit (Invitrogen) according to the manufacturer's instructions with a DNase treatment step. RNA concentration and purity was assessed using a Nanodrop (Thermofisher). RNA stability was verified by RNA Flash Gel (Lonza) to visualize $18 \mathrm{~S}$ and 28S rRNA bands. $1000 \mathrm{ng}$ of total RNA was added to each $20 \mu \mathrm{L}$ cDNA synthesis reaction, which was reverse transcribed using iScript Reverse Transcription Supermix (BioRad). Primer sequences, annealing temperatures, product sizes and assay validation were previously described (15). 
Table 1: Primer sequences and amplicon size for sex determination assay from (14).

\begin{tabular}{ccc}
\hline Primer & Primer Sequence & Amplicon Size (bp) \\
\hline$B$-Actin Forward & AGCCATGTACGTAGCCATCC & 220 \\
$B$-Actin Reverse & TGTGGTGGGAAGCTGTAGC & \\
$S R Y$ Forward & TACAGCCTGAGGACATATTA & 317 \\
$S R Y$ Reverse & CGACTTTAACCCTTCGATGA & \\
\hline
\end{tabular}

A



B

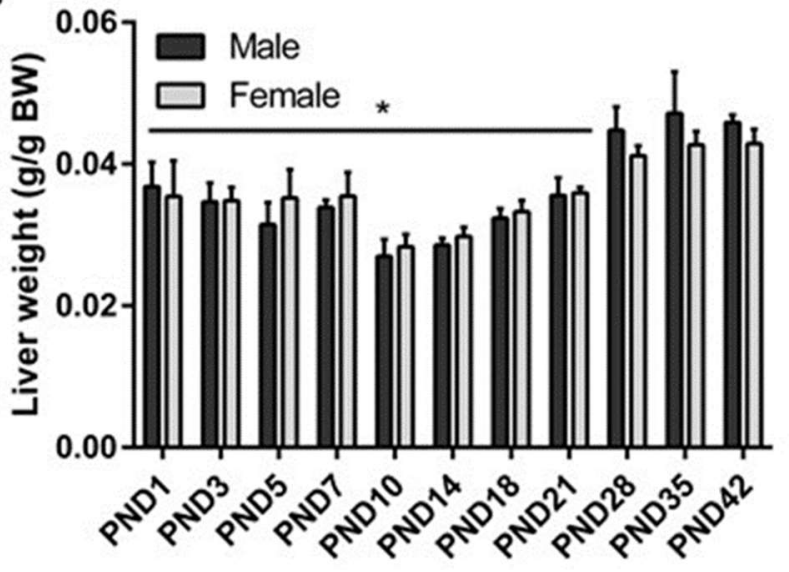

Figure 1: Body [A] and liver weights (normalized to body weight) [B] for male and female Sprague-Dawley rats from birth to sexual maturity. Data is presented as mean \pm $\mathrm{SD}(\mathrm{N}=4-5$ rats per age and sex $) .{ }^{*} \mathrm{P}<0.05$.

Serial dilutions of plasmids (covering 5 to 6 orders of magnitude) containing the gene-specific amplicon were used as standards to confirm qPCR efficiency and to quantify relative gene expression. Quantitative analyses of MCT1, MCT4, and CD147 were performed with iTaq Universal SYBR Green Supermix as described previously (15) on a CFX96 Connect (BioRad) with a standard curve run on every plate. The external control Alien RNA (Agilent) was utilized for data normalization. All biological samples were run in triplicate. The purity of all qPCR products was determined by melting curve. Data was quantified by $2^{-\Delta \Delta C T}$ method (16) or in arbitrary units based on the standard curve.

Western Blot Analysis: Whole cell extracts were generated from $25 \mathrm{mg}$ of rat liver tissue and $500 \mathrm{ul}$ of ice-cold radioimmunoprecipitation assay (RIPA) lysis buffer containing UltraCruz Protease Inhibitor Cocktail (Santa Cruz Biotechnology). Samples were briefly homogenized then spun at $13,500 \mathrm{rpm}$ in a $4^{\circ} \mathrm{C}$ microcentrifuge to collect protein supernatants. Soluble cytosolic and total membrane fractions were isolated from $25 \mathrm{mg}$ liver samples using a Calbiochem ProteoExtract Native Membrane Protein Extraction Kit (EMD-Millipore) following the manufacturer's instructions. Protein concentrations were calculated using the Pierce BCA Protein Assay kit (Thermo Fisher Scientific). MCT1, MCT4 and CD147 protein expression was quantified using western blot analysis of whole cell extract or membrane fraction. Samples $(10 \mu \mathrm{g}$ whole cell extract or $5 \mu \mathrm{g}$ membrane fraction) were mixed with 2x Laemmli sample buffer (BioRad) and heated at $37^{\circ} \mathrm{C}$ for 30 minutes, then loaded onto $10 \%$ TGX Fastcast acrylamide gels (Biorad) and separated via SDS-PAGE at $200 \mathrm{~V}$ for approximately 45 minutes. Following transfer onto nitrocellulose membranes at $100 \mathrm{~V}$ for 25 minutes, membranes were blocked in $5 \%$ nonfat milk in phosphate-buffered saline with Tween 20 (PBST) for 1 hour at ambient temperature, then incubated for 16 hours at $4^{\circ} \mathrm{C}$ under gentle rocking with anti-MCT1 (1:1350, EMD Millipore), anti-MCT4 (1:1000, Santa Cruz Biotechnology), anti-CD147 (1:5000, Santa Cruz Biotechnology), or anti-Na+/K+ ATPase (1:1000, Santa Cruz Biotechnology). All antibodies were formulated in 
$1 \%$ nonfat milk/PBST. After washing three times for 10 min in PBST, blots were incubated with goat antirabbit IgG horseradish peroxidase-conjugated (1:5000 Santa Cruz Biotechnology) or donkey antigoat IgG horseradish peroxidase-conjugated secondary antibodies (1:10000 EMD Millipore) for 1 hour at ambient temperature. Following a final $3 \times 10$ min PBST wash, immunoreactive bands were visualized using Clarity Western ECL substrate (Biorad) and band densities determined using ChemiDoc Touch Imaging System (Biorad).

Data Analysis: Data is presented as mean \pm standard deviation. Data was analyzed in GraphPad Prism 6 software using one-way analysis of variance (ANOVA), followed by Tukey's HSD post hoc test. Differences of $p<0.05$ in post-hoc comparison were considered to be significant. Pearson correlation analysis in GraphPad Prism 6 was used to evaluate the relationships between whole cell and membrane protein expression of MCT1, MCT4 and CD147.

\section{RESULTS}

\section{Sex Determination}

Sex was assigned at tissue collection by ano-genital distance for all pups less than PND21 with a target of four males and four females per age group. Based on PCR analysis of the SRY sequence, sex assignments were accurate for all pups at PND5 and later (data not shown). At PND1, the final PCR sex assignments yielded three male and five female pups (Figure 2).

\section{MCT1 Expression}

Males and females had similar nonlinear transcriptional MCT1 profiles during development (Figure $3 \mathrm{a}$ and $3 \mathrm{~b}$ ). Expression generally increased with age, with peak mRNA levels at PND10 in the males and PND 14 in females, before decreasing incrementally towards PND42. In males (Figure 3c), MCT1 whole cell protein expression was lowest at PND1, before increasing gradually with the highest expression observed at PND14, before reverting to expression levels consistent with neonatal animals at adolescence (PND42). A similar trend was observed in the females (Figure 3d), although expression was higher at PND1 and lower at PND42 as compared to the males. Similar patterns were observed for MCT1 membrane expression (Figure $3 \mathrm{e}$ and $3 \mathrm{f}$ ), with reduced expression at PND1 and PND42, and significantly higher expression at PND14 and PND18 in the males and females, with the males also having significantly increased expression at PND10.

\section{MCT4 Expression}

Males and females had similar linear transcriptional MCT4 profiles during development (Figure 4a and $4 \mathrm{~b}$ ), with the highest levels in neonatal rats at PND1, 3,5 , and 7 , before decreasing incrementally with age towards PND42 (adolescents). This differed from the observed patterns for MCT1 suggesting transporterspecific developmental regulation. MCT4 expression was undetectable in whole cell protein samples over the age range evaluated for both sexes. In males and females, membrane protein was minimally expressed at PND1, before increasing incrementally to peak at PND35, before decreasing drastically at PND42 (Figure 4c and 4d).



Figure 2: PCR confirmation of sex assignments by ano-genital distance. Genomic DNA was isolated from the liver and SRY (317 bp) was utilized to confirm the presence of the Y chromosome. B-actin (220 bp) served as a PCR control, and PND 21 animals served as positive controls for sex assignments. 


\section{CD147 Expression}

Males and females demonstrated nonlinear developmental expression patterns for both whole cell and membrane protein. In males, CD147 whole protein expression was low at PND1 and increased to peak levels at PND14 and PND18 (approximately three times higher expression), before experiencing a decrease in expression levels towards PND42 (Figure 5a). In females, CD147 expression was low at PND1, before increasing significantly to its highest levels at PND14, before decreasing again to neonatal levels at PND42 (Figure 5b). While males and females had similar expression levels at PND1, males had higher whole cell CD147 expression at PND42 compared to PND1, and to females at PND42. Membrane protein expression demonstrated similar expression pattern in both males and females (Figures 5b and 5c). Males demonstrated the highest membrane CD147 expression at PND14, while females demonstrated significantly higher expression at PND10, PND14 and PND18. Consistent with whole cell CD147 expression at PND42, males demonstrated approximately 2.5 times higher CD 147 membrane expression as compared to females.

\section{Correlation Analysis}

In the male ontogeny (Figure 6a), comparisons between membrane and whole cell MCT1 expression displayed a significant positive correlation with an $r$ value of 0.545 . The female ontogeny (Figure 6b) had a similar significant positive correlation with an $\mathrm{r}$ value of 0.587 . Correlations on the relationship between MCT1 and CD147 membrane and whole cell protein expression were conducted to investigate membrane trafficking. In males (Figure 6c), there was a significant positive correlation with an $r$ value of 0.585 , while females (Figure 6d) had a significant positive correlation; however, the $r$ value $(0.342)$ was lower than that observed in males. Correlation analysis between membrane expression of MCT1 and CD147 demonstrated strong significant positive correlations with $\mathrm{r}$ values of 0.8318 and 0.8447 in males and females (Figure 6e and 6f). Relationships between
MCT4 whole cell and membrane expression could not be evaluated, as whole cell MCT4 expression was not detectable. Correlation analysis was conducted to evaluate the trafficking relationship between MCT4 and CD147 membrane expression. In contrast to MCT1, MCT4 membrane expression demonstrated a weak negative correlation with membrane CD147 expression; however, this relationship was not significant in male or females (Figure $7 \mathrm{a}$ and $7 b$ ).

\section{DISCUSSION}

Monocarboxylate transporters are ubiquitously expressed in the human body, and studies have shown MCTs are associated with the absorption and excretion of monocarboxylate drugs in drug disposition organs such as the liver, kidneys, and intestines. The extent of transport capacity is dependent on MCT membrane protein expression, with changes in expression altering drug disposition in cells and tissues. However, there is limited data regarding the maturation and expression of hepatic MCTs relative to age, particularly during pediatric development in both males and females. A technical obstacle in clinical studies is that hepatic fetal and infant tissues are often derived postmortem, with confounding factors such as race, sex, disease, and polygenic traits, as well as limited sampling size presenting barriers to controlled pediatric studies $(13,17)$. To our knowledge, the present work is the first analysis of changes in rat hepatic MCT1, MCT4 and CD147 expression at the mRNA, whole cell, and membrane protein level during early stages of mammalian growth. Here, we show hepatic MCT1 and CD147 expression changes in a nonlinear fashion from birth to sexual maturity with a significant positive correlation between MCT1 and CD147 membrane expression. The maturation pattern of MCT4 differed from MCT1, with low expressions at birth followed by a surge in membrane expression at PND35. Further, there was a weak negative correlation between MCT4 and CD147 membrane expression. 
A


$\mathrm{Na}^{+} / \mathrm{K}^{+}$ATPase $-\cdots-m-m-m$


B


Figure 3: MCT1 mRNA, whole cell and membrane protein expression in males $[A, C, E]$ and females $[B, D, F]$ from birth to sexual maturity. $A, B$. Fold-change in MCT1 mRNA expression in rat liver. $C, D$. Whole cell MCT1 protein expression in the liver with data normalized to $\mathrm{Na}^{+} / \mathrm{K}^{+}$ATPase expression. A positive control sample (pooled liver whole cell sample from 9 week old male Sprague-Dawley rats) was loaded on each blot. E, F. MCT1 membrane expression in rat liver with data normalized to $\mathrm{Na}^{+} / \mathrm{K}^{+}$ATPase expression. A positive control sample (pooled liver whole cell sample from 9 week old male Sprague-Dawley rats) was loaded on each blot. Data is presented as mean $\pm \mathrm{SD}(\mathrm{N}=4-5$ rats per age and sex). $* \mathrm{P}<0.05$ 



Figure 4: MCT4 mRNA, whole cell and membrane protein expression in males $[A, C]$ and females $[B, D]$ from birth to sexual maturity. $A, B$. Fold-change in MCT4 mRNA expression in rat liver relative to PND21 females. $C, D$. MCT4 membrane expression in rat liver with data normalized to $\mathrm{Na}^{+} / \mathrm{K}^{+}$ATPase expression. A positive control sample (pooled liver whole cell sample from 9 week old male Sprague-Dawley rats) was loaded on each blot. Data is presented as mean $\pm \mathrm{SD}(\mathrm{N}=4-5$ rats per age and sex). $* \mathrm{P}<0.05$.

MCT1 is responsible for nutrient transport and essential for the metabolism of carbohydrates, amino acids, and fats. Consequently, dysregulation of MCT1 expression is associated with metabolic disorders such as obesity and abnormal insulin secretion (18), as well as altered drug transport and pharmacokinetics. MCT1 expression is detected as early as oocyte maturation (19), and knockout of the transporter leads to embryonic lethality in mouse animal models, indicating its critical role in early mammalian development and survival (20). Comparisons between human fetal (gestational age range of 16.4-37.9 weeks), infant (postnatal age range of 0-11.4 weeks, gestational age at birth range of 27.1-41 weeks), and adult specimen demonstrated nonlinear maturation of MCT1 membrane protein in the liver (13), which is consistent with that pattern of MCT1 maturation observed in the present study. Maturation of mRNA and whole cell protein expression of MCT1 was consistent with membrane protein expression; however, the moderate positive correlation suggests that MCT1 translation does not completely account for the transporter's expression at the plasma membrane suggesting that other regulatory factors mediate trafficking of MCT1 to the cell membrane during development. 




Figure 5: $\mathrm{CD} 147$ cell and membrane protein expression in males $[A, C]$ and females $[B, D]$ from birth to sexual maturity. $A$, $B$. Whole cell CD147 protein expression in the liver with data normalized to $\mathrm{Na}^{+} / \mathrm{K}^{+}$ATPase expression. A positive control sample (pooled liver whole cell sample from 9 week old male Sprague-Dawley rats) was loaded on each blot. C, D. CD147 membrane expression in rat liver with data normalized to $\mathrm{Na}^{+} / \mathrm{K}^{+}$ATPase expression. A positive control sample (pooled liver whole cell sample from 9 week old male Sprague-Dawley rats) was loaded on each blot. Data is presented as mean $\pm \mathrm{SD}(\mathrm{N}$ $=4-5$ rats per age and sex). $* \mathrm{P}<0.05$.

Developmental mRNA expression of MCT4 diverges from the MCT1 profile, with high mRNA expression at birth and decreasing levels towards sexual maturity; however, MCT4 protein data reverses this trend, with low membrane protein expression at early ages until a sudden peak at postnatal age 35 . The mechanisms underlying this discrepancy between MCT4 mRNA and protein expression are not completely understood. Other reviews have reported the historically poor correlation observed between mRNA and ex-vivo protein in complex biological samples, which complicates data interpretation for transcriptionaltranslational model predictions (21). Previous studies have shown that endogenous microRNAs, miR-29 and miR-124, can degrade MCT1 mRNA or inhibit its translation, while let-b7 downregulates CD147 expression (22), similar mechanisms could explain the discrepancy that we observed between
MCT4 mRNA and protein expression. miR-29 in particular is highly expressed in $\beta$ islet cells, which may explain the diminished amount of MCT1 found in pancreas compared to other tissues (23), and MCT regulation by miRNA may explain the observed tissue differences in expression. While fewer regulation studies have been performed on MCT4, the transcription factor HIF-1 $\alpha$ has been shown to upregulate mRNA and protein expression of MCT4 during hypoxia, but not MCT1 (24). However, the mechanisms regulating MCT4 expression under normoxic conditions remain to be determined (25). Further experiments identifying possible miRNA activity and other post-transcriptional modifications may explain the dynamic changes in MCT1 and MCT4 expression based on maturation, with subsequent upregulation and downregulation events dependent on MCT isoform, biological sample, and tissue-type (26). 
A

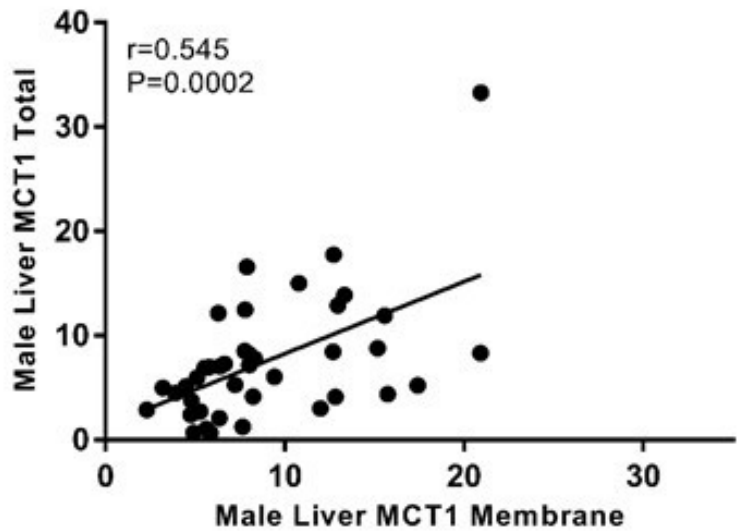

C



E



B



D



$\mathbf{F}$

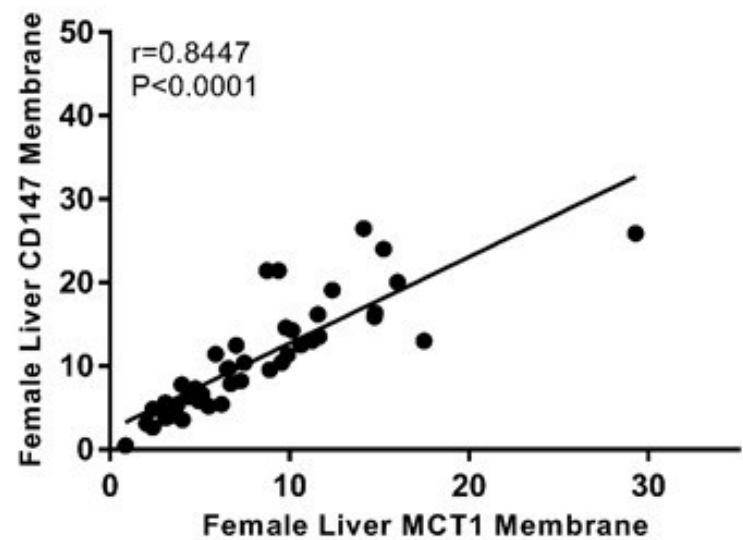

Figure 6: Pearson correlation analysis in males $[A, C, E]$ and females $[B, D, F] . A, B$. Correlation analysis between MCT1 whole cell protein and membrane expression. $C, D$. Correlation analysis between whole cell MCT1 and CD147 expression. $E, F$. Correlation analysis between membrane MCT1 and CD147 expression. $(\mathrm{N}=42$ males; $\mathrm{N}=44$ females) 




B

Figure 7: Pearson correlation analysis between membrane MCT4 and CD147 expression in males [A] and females [B]. (N $=42$ males; $\mathrm{N}=44$ females)

Unlike hepatic transporters and drug metabolizing enzymes, few studies have looked at the age-dependent expression of ancillary proteins in the liver, or their mechanistic roles in drug transporter maturation. The CD147 glycoprotein binds to MCT1 and MCT4 and confers basolateral sorting onto the heterodimer, facilitating the transporter's localization to the plasma membrane where it becomes functionally active (27). Studies have shown that increased association between MCT1/4 and CD147 enhances plasma membrane expression of the transporter (28), whereas knockdown or silencing of the chaperone in various cells lines and transgenic animal models causes decreased MCT1/CD147 association and membrane co-localization, as well as overall altered tissue distribution (29). CD147 in the rumen of juvenile sheep was found to increase linearly based on age (30). In our experiment, whole cell CD147 protein is expressed at minimal levels at birth, with increasing and maximum levels at postnatal ages 14/18 in the males and postnatal age 14 in females, then decreasing towards neonatal levels upon sexual maturity. Membrane levels of CD147 reflect the total protein trend, with peak expressions at postnatal age 14 in both sexes, and there exists strong, positive correlation between total and membrane-bound CD147 protein expression. Comparison of total protein MCT1 and CD147 yielded a significant moderate correlation, indicating total translation of the ancillary protein has minimal impact on whole protein expression of its transporter partner. However, we observed a significant strong positive



correlation when comparing the membrane expression of MCT1 and CD147, signifying a strong association between the membrane proteins, and suggesting the ancillary protein is required as a chaperone for proper MCT1 trafficking to the cell surface throughout normal mammalian development, with a nonlinear expressional pattern dependent on age. In contrast, MCT4 membrane expression demonstrated a weak negative correlation with CD147 membrane expression suggesting that CD147 does not determine MCT4 membrane expression during development. The present study did not evaluate MCT4 membrane localization, which may be altered if it is no longer associated with CD147.

Previously, our group described the sex hormone dependent regulation and localization of MCT1 and MCT4 in the liver, with changes in expression between male and female rats, as well as in female rats at various stages in their estrus cycle (15). Consistent with our previous results, we observed variability in hepatic MCT1, MCT4, and CD147 expression between the males and females in both juvenile and sexually mature rats. Males exhibited increased MCT1 membrane expression at PND10 and 18 compared to the females. Similarly, MCT4 and CD147 membrane protein expression was higher in males compared to the females in juvenile rats, implicating a sex-specific, sex-hormone independent regulatory mechanisms in MCT expression. MCT1, MCT4 and CD147 membrane expression was higher in males than females at PND42, at which age rats will have circulating sex hormones. Increased 
abundance of MCTs and ancillary protein in males may result in greater hepatic transport capacity for nutrients and pharmaceutical agents compared to females, which can lead to increased drug absorption and reduced hepatobiliary clearance.

Our data is consistent with previous clinical studies on MCT maturation (13) and suggests several avenues for future transporter work. The nonlinearity of hepatic MCT1 expression indicates a clear need to assess the maturation of drug disposition processes to account for inter-individual variability in pharmacokinetics in pediatric populations. Drug uptake and efflux assays in the liver can also evaluate the mechanisms between MCT expression and function during development, as well as between adolescent male and female populations. Furthermore, we can explore the effects of age on the expressional changes of MCTs in other drug disposition tissues such as the intestines and kidney. In conclusion, we have demonstrated differential expression of hepatic MCT1, MCT4, and CD147 during development at the level of mRNA transcription, protein translation, and membrane trafficking in a pediatric animal model.

\section{ACKNOWLEDGEMENTS}

Funding support was provided by University of the Pacific through an FRC-SAAG award and start-up funding for MAF.

\section{REFERENCES}

1. Allegaert $\mathrm{K}$, Anderson $\mathrm{BJ}$, van den Anker JN, Vanhaesebrouck S, de Zegher F. Renal drug clearance in preterm neonates: relation to prenatal growth. Therapeutic drug monitoring. 2007;29(3):284-91. Epub 2007/05/29. doi: 10.1097/FTD.0b013e31806db3f5. PubMed PMID: 17529884.

2. Chen N, Aleksa K, Woodland C, Rieder M, Koren G. Ontogeny of drug elimination by the human kidney. Pediatr Nephrol. 2006;21(2):160-8. Epub 2005/12/07. doi: 10.1007/s00467-005-2105-4. PubMed PMID: 16331517.

3. Hines RN. The ontogeny of drug metabolism enzymes and implications for adverse drug events. Pharmacol Ther. 2008;118(2):250-67. Epub 2008/04/15. doi: 10.1016/j.pharmthera.2008.02.005. PubMed PMID: 18406467.

4. Elmorsi Y, Barber J, Rostami-Hodjegan A. Ontogeny of Hepatic Drug Transporters and Relevance to Drugs Used in Pediatrics. Drug Metab Dispos. 2016;44(7):992-8. Epub 2015/12/30. doi: 10.1124/dmd.115.067801. PubMed PMID: 26712821.

5. Morris ME, Felmlee MA. Overview of the protoncoupled MCT (SLC16A) family of transporters: characterization, function and role in the transport of the drug of abuse gamma-hydroxybutyric acid. AAPS J. 2008;10(2):311-21. Epub 2008/06/05. doi: 10.1208/s12248-008-9035-6. PubMed PMID: 18523892; PMCID: PMC2574616.

6. Kohyama N, Shiokawa H, Ohbayashi M, Kobayashi Y, Yamamoto T. Characterization of monocarboxylate transporter 6: expression in human intestine and transport of the antidiabetic drug nateglinide. Drug Metab Dispos. 2013;41(11):18837. Epub 2013/08/13. doi: 10.1124/dmd.113.051854. PubMed PMID: 23935065.

7. Bhattacharya I, Boje KM. GHB (gammahydroxybutyrate) carrier-mediated transport across the blood-brain barrier. J Pharmacol Exp Ther. 2004;311(1):92-8. doi: 10.1124/jpet.104.069682. PubMed PMID: 15173314.

8. Ganapathy V, Thangaraju M, Gopal E, Martin PM, Itagaki S, Miyauchi S, Prasad PD. Sodium-coupled monocarboxylate transporters in normal tissues and in cancer. AAPS J. 2008;10(1):193-9. doi: 10.1208/s12248-008-9022-y. PubMed PMID: 18446519; PMCID: 2751467.

9. Iwanaga $\mathrm{T}$, Kishimoto A. Cellular distributions of monocarboxylate transporters: a review. Biomed Res. 2015;36(5):279-301. Epub 2015/11/03. doi: 10.2220/biomedres.36.279. PubMed PMID: 26522146.

10. Morse BL, Felmlee MA, Morris ME. gammaHydroxybutyrate blood/plasma partitioning: effect of physiologic $\mathrm{pH}$ on transport by monocarboxylate transporters. Drug Metab Dispos. 2012;40(1):64-9. doi: 10.1124/dmd.111.041285. PubMed PMID: 21976619; PMCID: 3250051.

11. Kirk P, Wilson MC, Heddle C, Brown MH, Barclay AN, Halestrap AP. CD147 is tightly associated with lactate transporters MCT1 and MCT4 and facilitates their cell surface expression. EMBO J. 2000;19(15):3896-904. Epub 2000/08/02. doi: 10.1093/emboj/19.15.3896. PubMed PMID: 10921872; PMCID: PMC306613.

12. Halestrap AP, Price NT. The proton-linked monocarboxylate transporter (MCT) family: structure, function and regulation. Biochem $\mathrm{J}$. 1999;343 Pt 2:281-99. PubMed PMID: 10510291; PMCID: 1220552.

13. Mooij MG, Nies AT, Knibbe CA, Schaeffeler E, Tibboel D, Schwab M, de Wildt SN. Development of Human Membrane Transporters: Drug Disposition and Pharmacogenetics. Clin Pharmacokinet. 2016;55(5):507-24. Epub 2015/09/28. doi: 10.1007/s40262-015-0328-5. PubMed PMID: 26410689 ; PMCID: PMC4823323. 
14. Miyajima A, Sunouchi M, Mitsunaga K, Yamakoshi $Y$, Nakazawa K, Usami M. Sexing of postimplantation rat embryos in stored twodimensional electrophoresis (2-DE) samples by polymerase chain reaction (PCR) of an Sry sequence. The Journal Of Toxicological Sciences. 2009;34(6):681-5. PubMed PMID: 19952503.

15. Cao J, Ng M, Felmlee MA. Sex Hormones Regulate Rat Hepatic Monocarboxylate Transporter Expression and Membrane Trafficking. J Pharm Pharm Sci. 2017;20(1):435-44. doi: 10.18433/J3CH29. PubMed PMID: 29249221.

16. Livak KJ, Schmittgen TD. Analysis of relative gene expression data using real-time quantitative PCR and the 2(-Delta Delta C(T)) Method. Methods. 2001;25(4):402-8. Epub 2002/02/16. doi: 10.1006/meth.2001.1262. PubMed PMID: 11846609.

17. Brouwer KL, Aleksunes LM, Brandys B, Giacoia GP, Knipp G, Lukacova V, Meibohm B, Nigam SK, Rieder M, de Wildt SN, Pediatric Transporter Working G. Human Ontogeny of Drug Transporters: Review and Recommendations of the Pediatric Transporter Working Group. Clin Pharmacol Ther. 2015;98(3):266-87. Epub 2015/06/20. doi: 10.1002/cpt.176. PubMed PMID: 26088472; PMCID: PMC4731327.

18. Perez de Heredia F, Wood IS, Trayhurn P. Hypoxia stimulates lactate release and modulates monocarboxylate transporter (MCT1, MCT2, and MCT4) expression in human adipocytes. Pflugers Arch. 2010;459(3):509-18. Epub 2009/10/31. doi: 10.1007/s00424-009-0750-3. PubMed PMID: 19876643.

19. Vannucci SJ, Simpson IA. Developmental switch in brain nutrient transporter expression in the rat. Am J Physiol Endocrinol Metab. 2003;285(5):E1127-34. Epub 2003/10/10. doi: 10.1152/ajpendo.00187.2003. PubMed PMID: 14534079.

20. Lengacher S, Nehiri-Sitayeb T, Steiner N, Carneiro L, Favrod C, Preitner F, Thorens B, Stehle JC, Dix L, Pralong F, Magistretti PJ, Pellerin L. Resistance to diet-induced obesity and associated metabolic perturbations in haploinsufficient monocarboxylate transporter 1 mice. PLoS One. 2013;8(12):e82505. Epub 2013/12/25. doi: 10.1371/journal.pone.0082505. PubMed PMID: 24367518; PMCID: PMC3867350.

21. Maier T, Guell M, Serrano L. Correlation of mRNA and protein in complex biological samples. FEBS Lett. 2009;583(24):3966-73. Epub 2009/10/24. doi: 10.1016/j.febslet.2009.10.036. PubMed PMID: 19850042.

22. Hatziapostolou M, Polytarchou C, Iliopoulos D. miRNAs link metabolic reprogramming to oncogenesis. Trends Endocrinol Metab.
2013;24(7):361-73. Epub 2013/04/23. doi: 10.1016/j.tem.2013.03.002. PubMed PMID: 23602813.

23. Pullen TJ, da Silva Xavier G, Kelsey G, Rutter GA. miR-29a and miR-29b contribute to pancreatic betacell-specific silencing of monocarboxylate transporter 1 (Mct1). Mol Cell Biol. 2011;31(15):3182-94. Epub 2011/06/08. doi: 10.1128/MCB.01433-10. PubMed PMID: 21646425; PMCID: PMC3147603.

24. Ullah MS, Davies AJ, Halestrap AP. The plasma membrane lactate transporter MCT4, but not MCT1, is up-regulated by hypoxia through a HIF-1alphadependent mechanism. J Biol Chem. 2006;281(14):9030-7. Epub 2006/02/03. doi: 10.1074/jbc.M511397200. PubMed PMID: 16452478 .

25. Halestrap AP. The SLC16 gene family - structure, role and regulation in health and disease. Mol Aspects Med. 2013;34(2-3):337-49. Epub 2013/03/20. doi: 10.1016/j.mam.2012.05.003. PubMed PMID: 23506875.

26. Burgess KS, Philips S, Benson EA, Desta Z, Gaedigk A, Gaedigk R, Segar MW, Liu Y, Skaar TC. AgeRelated Changes in MicroRNA Expression and Pharmacogenes in Human Liver. Clin Pharmacol Ther. 2015;98(2):205-15. Epub 2015/05/15. doi: 10.1002/cpt.145. PubMed PMID: 25968989; PMCID: PMC4512918.

27. Castorino JJ, Deborde S, Deora A, Schreiner R, Gallagher-Colombo SM, Rodriguez-Boulan E, Philp NJ. Basolateral sorting signals regulating tissuespecific polarity of heteromeric monocarboxylate transporters in epithelia. Traffic. 2011;12(4):483-98. doi: 10.1111/j.1600-0854.2010.01155.x. PubMed PMID: 21199217; PMCID: 3132080.

28. Mannowetz N, Wandernoth P, Wennemuth G. Basigin interacts with both MCT1 and MCT2 in murine spermatozoa. J Cell Physiol. 2012;227(5):2154-62. Epub 2011/07/28. doi: 10.1002/jcp.22949. PubMed PMID: 21792931.

29. Nakai M, Chen L, Nowak RA. Tissue distribution of basigin and monocarboxylate transporter 1 in the adult male mouse: a study using the wild-type and basigin gene knockout mice. Anat Rec A Discov Mol Cell Evol Biol. 2006;288(5):527-35. Epub 2006/04/14. doi: 10.1002/ar.a.20320. PubMed PMID: 16612830; PMCID: PMC3739424.

30. Koho NM, Taponen J, Tiihonen H, Manninen M, Poso AR. Effects of age and concentrate feeding on the expression of MCT 1 and CD147 in the gastrointestinal tract of goats and Hereford finishing beef bulls. Res Vet Sci. 2011;90(2):301-5. Epub 2010/07/06. doi: 10.1016/j.rvsc.2010.06.002. PubMed PMID: 20598330. 